\title{
Cooperative distributed MPC for tracking
}

\author{
A. Ferramosca ${ }^{*}$ D. Limon ${ }^{*}$ J.B. Rawlings ${ }^{* *}$ E.F. Camacho* \\ * Departamento de Ingeniería de Sistemas y Automática, Universidad de \\ Sevilla, Escuela Superior de Ingenieros, Camino de los Descubrimientos $s / n$. \\ 41092 Sevilla, SPAIN (e-mail: \{ferramosca,limon,eduardo\}@cartuja.us.es) \\ ** Department of Chemical and Biological Engineering, University of \\ Wisconsin. Madison, WI. 53706-1691. USA (e-mail: \\ rawlings@engr.wisc.edu)
}

\begin{abstract}
The problem of controlling large scale systems, usually divided into subsystems controlled by different agents, is usually solved using cooperative distributed control schemes, where the agents share open-loop information in order to improve closed-loop performance, (Rawlings and Mayne 2009, Chapter 6). In this paper we proposed a cooperative distributed linear model predictive control strategy applicable to any finite number of subsystems, which is able to steer the system to any admissible set point in an admissible way. Feasibility is ensured under any changing of the target steady state. The proposed controller is applied to a four tanks system.
\end{abstract}

Keywords: model predictive control, distributed control, setpoint tracking.

\section{INTRODUCTION}

Model predictive control (MPC) is one of the most successful techniques of advanced control in the process industries. It is based on the solution of an optimization problem and allows to explicit consideration of hard constraints in the formulation (Camacho and Bordons 2004). Furthermore, asymptotic stability and constraints satisfaction of the closed-loop system has been established (Mayne et al. 2000, Rawlings and Mayne 2009, Chapter 2).

Large scale control systems usually consist of linked unit of operations and can be divided into a number of subsystems controlled by different agents which may or may not share information. A first approach to this problem is decentralized control, in which interactions between the different subsystems are not considered (Sandell Jr. et al. 1978). The main issue of this solution appears when the intersubsystem interactions become strong. Centralized control, in which a single agent controls the plantwide system, is another traditional solution that can cope with this control problem. The main problems of this solution are the computational burden and the coordination of subsystems and controller. Distributed control schemes, where agents share open-loop information in order to improve closedloop performance, solve many of these problems (Rawlings and Mayne 2009, Chapter 6).

The difference between the distributed control strategies is in the use of this open-loop information. In noncooperative distributed control each subsystem considers the other subsystems information as a known disturbance (Camponogara et al. 2002, Dunbar 2007). This strategy leads the overall system to converge to a Nash equilibrium. In cooperative distributed control the agents share a common objective and optimize a cost function that can be considered as the overall system cost function (Venkat 2006). This strategy is a form of suboptimal control: stability is deduced from suboptimal control theory (Scokaert et al. 1999) and convergence to a Pareto optimum is ensured. MPC is one of the most used control structure to cope with distributed control. In (Magni and Scattolini 2006) an MPC approach for nonlinear systems is proposed, where no information is exchanged between the local controllers. An inputto-state stability proof for this approach is given in (Raimondo et al. 2007). In (Liu et al. 2009, 2008) the authors present a controller for networked nonlinear systems, which is based on a Lyapunov-based model predictive control. In (Venkat et al. 2007) a cooperative distributed MPC is presented, in which suboptimal input trajectories are used to stabilize the plant. Most of the results on MPC consider the regulation problem, that is steering the system to a fixed setpoint. When this setpoint changes, the feasibility of the controller may be lost and the controller may fail to track the reference (Rao and Rawlings 1999, Pannocchia and Kerrigan 2005, Rossiter et al. 1996, Bemporad et al. 1997). In (Limon et al. 2008, Ferramosca et al. 2009) an MPC for tracking of constrained linear systems is proposed, which is able to lead the system to any admissible setpoint, maintaining feasibility. The main characteristics of this controller are: an artificial steady state considered as a decision variable, a cost that penalizes the error with the artificial steady state, an additional term that penalizes the deviation between the artificial steady state and the target steady state added to the cost function (the offset cost function) and an invariant set for tracking considered as an extended terminal constraint. In this paper, this controller is extended to the case of distributed systems, considered as a cooperative game, and it is applied to a four tanks system.

The paper is organized as follows. In section 2 the constrained tracking problem is stated. In section 3 the proposed cooperative distributed MPC for tracking is presented. In section 4 an illustrative example is shown. Finally, the conclusions of this work are given in section 5 .

\section{PROBLEM STATEMENT}

Consider a system described by a linear invariant discrete time model 


$$
\begin{aligned}
x^{+} & =A x+B u \\
y & =C x+D u
\end{aligned}
$$

where $x \in \mathbb{R}^{n}$ is the system state, $u \in \mathbb{R}^{m}$ is the current control vector, $y \in \mathbb{R}^{p}$ is the controlled output and $x^{+}$is the successor state. The solution of this system for a given sequence of control inputs $\mathbf{u}$ and initial state $x$ is denoted as $x(j)=\phi(j ; x, \mathbf{u})$ where $x=\phi(0 ; x, \mathbf{u})$. The state of the system and the control input applied at sampling time $k$ are denoted as $x(k)$ and $u(k)$ respectively. The system is subject to hard constraints on state and control:

$$
x(k) \in X, \quad u(k) \in U
$$

for all $k \geq 0 . X \subset \mathbb{R}^{n}$ and $U \subset \mathbb{R}^{m}$ are compact convex polyhedra containing the origin in their interiors. It is assumed that the following hypothesis hold.

Assumption 1. The pair (A,B) is stabilizable and the state is measured at each sampling time.

The steady state, input and output of the plant $\left(x_{s}, u_{s}, y_{s}\right)$ are such that (1) is fulfilled, i.e. $x_{s}=A x_{s}+B u_{s}$, and $y_{s}=C x_{s}+$ $D u_{s}$.

We define the sets of admissible equilibrium states, inputs and outputs as

$$
\begin{aligned}
& z_{s}=\{(x, u) \in X \times U \mid x=A x+B u\} \\
& x_{s}=\left\{x \in X \mid \exists u \in U \text { such that }(x, u) \in z_{s}\right\} \\
& y_{s}=\left\{y=C_{x}+D_{u} \mid(x, u) \in z_{s}\right\}
\end{aligned}
$$

Notice that $X_{s}$ is the projection of $Z_{s}$ onto $X$.

Under assumption 1 , given a steady output $y_{s}$, any steady state and input of system (1) associated to this output must satisfy the following equation:

$$
\left[\begin{array}{ccc}
A-I_{n} & B & \mathbf{0}_{p, 1} \\
C & D & -I_{p}
\end{array}\right]\left[\begin{array}{l}
x_{s} \\
u_{s} \\
y_{s}
\end{array}\right]=\left[\begin{array}{l}
\mathbf{0}_{n, 1} \\
\mathbf{0}_{p, 1}
\end{array}\right]
$$

In (Limon et al. 2008) the authors state that the steady state and input $\left(x_{s}, u_{s}\right)$ of the system can be parameterized as a linear combination of a vector $\theta \in \mathbb{R}^{m}$. In this paper, we choose a steady output $y_{s}$ to parameterize the couple $\left(x_{s}, u_{s}\right)$, in such a way that there exists a suitable matrix $M_{y}$ such that $\left(x_{s}, u_{s}\right)=M_{y} y_{s}$. This parametrization is possible if and only if Lemma 1.14 in (Rawlings and Mayne 2009, p. 83) holds. If this condition does not hold, the parametrization presented in (Limon et al. 2008) has to be used.

In this paper, a decentralized control framework is considered. Thus, it is assumed that system (1) can be partitioned in $M$ subsystems of the form (Rawlings and Mayne 2009, Chapter 6, pp. 421-422):

$$
x_{i}^{+}=A_{i} x_{i}+\sum_{j=1}^{M} \bar{B}_{i j} u_{j}
$$

where $x_{i} \in \mathbb{R}^{n_{i}}, u_{j} \in \mathbb{R}^{m_{j}}, A_{i} \in \mathbb{R}^{n_{i} \times n_{i}}$ and $B_{i j} \in$ $\mathbb{R}^{n_{i} \times m_{j}}$. A discussion on the identification of these models is given in (Gudi and Rawlings 2006) while in (Stewart et al. 2010, Appendix B) is shown how these models can be derived from any centralized model. As for the output, the model is given by:

$$
y_{i}=C_{i} x_{i}+\sum_{j=1}^{M} \bar{D}_{i j} u_{j}
$$

with $y_{i} \in \mathbb{R}_{i}^{p}, C_{i} \in \mathbb{R}^{p_{i} \times n_{i}}$ and $D_{i j} \in \mathbb{R}^{p_{i} \times m_{j}}$. In this case, some observability assumptions are necessary.

For the sake of simplicity of the exposition, the results will be presented for the case of a two player game. In this case, the plant can be represented in the form:

$$
\left[\begin{array}{l}
x_{1} \\
x_{2}
\end{array}\right]^{+}=\left[\begin{array}{ll}
A_{1} & \\
& A_{2}
\end{array}\right]\left[\begin{array}{l}
x_{1} \\
x_{2}
\end{array}\right]+\left[\begin{array}{l}
\bar{B}_{11} \\
\bar{B}_{21}
\end{array}\right] u_{1}+\left[\begin{array}{l}
\bar{B}_{12} \\
\bar{B}_{22}
\end{array}\right] u_{2}
$$

The solution of this system, given the sequences of control inputs $\mathbf{u}_{1}$ and $\mathbf{u}_{2}$ and initial state $x$ is denoted as $x(j)=$ $\phi\left(j ; x, \mathbf{u}_{1}, \mathbf{u}_{2}\right)$ where $x=\phi\left(0 ; x, \mathbf{u}_{1}, \mathbf{u}_{2}\right)$. For the partitioned model of the plant, the steady state and input can be rewritten as

$$
\left(x_{s, i}, u_{s, i}\right)=M_{y_{i}} y_{s}
$$

The problem we consider is the design of a distributed MPC controller $u_{i}=\kappa_{i}\left(x, y_{t}\right)$ to track a (possible time-varying) plant-wide target output $y_{t}$, such that the subsystems are steered (as close as possible) to the target while fulfilling the constraints.

\section{COOPERATIVE MPC FOR TRACKING}

Among the existing solutions for the decentralized predictive control problem, we focus our attention on the cooperative game (Stewart et al. 2010, Rawlings and Mayne 2009, Chapter 6, p. 433). In cooperative distributed MPC, the two players share a common objective, which can be considered as the overall plant objective. Each $i$-th agent calculates its corresponding input $u_{i}$ by solving an iterative decentralized optimization problem. Stability is, therefore, proved by means of the theory of suboptimal MPC (Scokaert et al. 1999). It has been demonstrated that this approach ensures recursive feasibility, optimality (in case of uncoupled constraints) and asymptotic stability under mild assumptions. See (Rawlings and Mayne 2009, Chapter 6, pp. 446-453) and (Stewart et al. 2010) for a more detailed exposition.

In the problem of tracking non-zero setpoints, each agent has to solve a decentralized target problem at any iteration. In (Rawlings and Mayne 2009, Chapter 6, p. 458) the authors suggest, in case of coupled constraints, to solve a centralized target problem. When the target setpoint changes, the problem may become unfeasible, leading to the necessity of redesign the whole controller.

In this paper, a new cooperative distributed MPC for tracking is presented aimed to ensure convergence to the centralized optimal target without loosing feasibility after any change of the operation point. To this aim, as in the centralized case (Limon et al. 2008, Ferramosca et al. 2009), an artificial equilibrium point $\left(\hat{x}_{s}, \hat{u}_{s}, \hat{y}_{s}\right)$ is added as decision variable and the following modified cost function is considered:

$$
\begin{aligned}
V_{t}\left(x, y_{t} ; \mathbf{u}_{1}, \mathbf{u}_{2}, \hat{y}_{s}\right)= & \sum_{j=0}^{N-1}\left\|x(j)-\hat{x}_{s}\right\|_{Q}^{2}+\left\|u(j)-\hat{u}_{s}\right\|_{R}^{2} \\
& +\left\|x(N)-\hat{x}_{s}\right\|_{P}^{2}+V_{O}\left(\hat{y}_{s}-y_{t}\right)
\end{aligned}
$$

where $V_{O}\left(\hat{y}_{s}-y_{t}\right)$ is the so called offset cost function and is defined as follows:

Definition 1. Let $V_{O}\left(y-y_{t}\right)$ be a convex positive definite function such that the minimizer of

$$
\min _{y \in y_{s}} V_{O}\left(y-y_{t}\right)
$$


is unique and it is denoted as $y_{s}$, which is the optimal steady output of the centralized problem.

Typically this function is chosen as a norm of a distance (Ferramosca et al. 2009).

The following assumption is considered:

\section{Assumption 2.}

(1) Let $R_{i} \in \mathbb{R}^{m \times m}$ be a positive semidefinite matrix and $Q_{i} \in \mathbb{R}^{n \times n}$ a positive semi-definite matrix such that the pair $\left(Q_{i}^{1 / 2}, A_{i}\right)$ is observable.

(2) Let $K \in \mathbb{R}^{m \times n}$ be a stabilizing control gain for the centralized system, such that $(A+B K)$ is Hurwitz.

(3) Let $P \in \mathbb{R}^{n \times n}$ be a positive definite matrix for the centralized system such that:

$$
(A+B K)^{T} P(A+B K)-P=-\left(Q+K^{T} R K\right)
$$

(4) Let $\Omega_{t, K} \subseteq \mathbb{R}^{n+p}$ be an admissible polyhedral invariant set for tracking for system (1) subject to (2), for a given gain $K$ and the steady states set $\lambda X_{s}$, for any $\lambda \in(0,1)$. See Limon et al. (2008) for more details.

At each sampling time $k$ and iteration $p+1$, each subsystem $i \in \mathbb{I}_{[1,2]}$ solves the following optimization problem:

$$
\begin{aligned}
\left(\mathbf{u}_{i}^{0}, \hat{y}_{s, i}^{0}\right)= & \arg \min _{\mathbf{u}_{i}, \hat{y}_{s, i}} V_{t}\left(x, y_{t} ; \mathbf{u}_{1}, \mathbf{u}_{2}, \hat{y}_{s}\right) \\
\text { s.t. } & (8) \text { with } x(0)=\left(x_{1}, x_{2}\right), \\
& \mathbf{u}_{\ell}=\mathbf{u}_{\ell}^{[p]}, \quad \ell \in \mathbb{I}_{1,2} \backslash i, \\
& x \in X, \\
& \left(u_{1}(j), u_{2}(j)\right) \in U, \quad j=0, \ldots, N-1 \\
& \left(x(N), \hat{y}_{s}\right) \in \Omega_{t, K}
\end{aligned}
$$

Remark 2. At any sample time $k$ any $i$ agent computes its optimal control sequence $\mathbf{u}_{i}$ based on the state of the whole plant. The fact that each agent knows the overall system state is important for the satisfaction of the terminal constraint.

Remark 3. (Offset cost function). At any iteration, each agent solves the overall tracking problem, by finding a global $\hat{y}_{s}$.

Following (Stewart et al. 2010), the solution of the $p+1$ iteration is given by

$$
\begin{aligned}
\mathbf{u}_{1}^{[p+1]} & =w_{1} \mathbf{u}_{1}^{0}\left(x, \mathbf{u}_{2}^{[p]}\right)+w_{2} \mathbf{u}_{1}^{[p]} \\
\mathbf{u}_{2}^{[p+1]} & =w_{1} \mathbf{u}_{2}^{[p]}+w_{2} \mathbf{u}_{2}^{0}\left(x, \mathbf{u}_{1}^{[p]}\right) \\
\hat{y}_{s}^{[p+1]} & =w_{1} \hat{y}_{s, 1}^{0}\left(x, \mathbf{u}_{2}^{[p]}\right)+w_{2} \hat{y}_{s, 2}^{0}\left(x, \mathbf{u}_{1}^{[p]}\right) \\
w_{1}+w_{2} & =1 \quad w_{1}, w_{2}>0
\end{aligned}
$$

Therefore, as in (Stewart et al. 2010), at the last iteration $\bar{p}=$ $N_{\text {iter }}$, the solutions of the optimization problem are functions of the state and the optimal control sequences at time $k$ :

$$
\mathbf{u}(k+1)=g(x(k), \mathbf{u}(k))
$$

and it is given by $\mathbf{u}(k+1)=\left(\mathbf{u}_{1}^{[\bar{p}]}, \mathbf{u}_{2}^{[\bar{p}]}\right)$. The first element of the control sequence, $u(0)$, is injected into the plant.

Since distributed MPC control algorithms are based on suboptimal MPC (Stewart et al. 2010), at any time $k$ it is important to choose a feasible initialization of the optimization algorithm. This initialization, usually called the warm start plays an important role in suboptimal MPC, because it determines recursive feasibility and convergence of the control algorithm. In cooperative MPC for regulation (Stewart et al. 2010, Rawlings and Mayne 2009, Cap. 6), the warm start control sequence is obtained by discarding the first input, shifting the rest of the sequence forward one step and setting the last input to zero. In this paper we propose the following algorithm:

\section{Algorithm 1.}

Consider that at time $k+1$ the successor state of $x(k+1)$ has been computed using the optimal sequences $\mathbf{u}^{0}(x(k))^{1}$. Denote the vector of optimization variable as $\mathbf{v}=\left(\mathbf{u}_{1}, \mathbf{u}_{2}, \hat{y}_{s}^{0}\right)$.

- Define the first candidate initial solution:

$$
\begin{aligned}
& \tilde{\mathbf{u}}_{1}(k+1)=\left\{u_{1}^{0}(1), \ldots, u_{1}^{0}(N-1), u_{c, 1}^{0}(N)\right\} \\
& \tilde{\mathbf{u}}_{2}(k+1)=\left\{u_{2}^{0}(1), \ldots, u_{2}^{0}(N-1), u_{c, 2}^{0}(N)\right\}
\end{aligned}
$$

where $u_{c}^{0}(N)=\left(u_{c, 1}^{0}(N), u_{c, 2}^{0}(N)\right)$ and $u_{c}^{0}(N)=$ $K x^{0}(N)+L \hat{y}_{s}^{0}(k)$ is the centralized solution given by the centralized terminal control law, and $x^{0}(N)=$ $\phi\left(N ; \mathbf{u}_{1}^{0}, \mathbf{u}_{2}^{0}\right)$.

- Define the second candidate initial solution:

$$
\begin{aligned}
& \hat{\mathbf{u}}_{1}(k+1)=\left\{\hat{u}_{c, 1}^{0}(0), \ldots, \hat{u}_{c, 1}^{0}(N-1)\right\} \\
& \hat{\mathbf{u}}_{2}(k+1)=\left\{\hat{u}_{c, 2}^{0}(0), \ldots, \hat{u}_{c, 2}^{0}(N-1)\right\}
\end{aligned}
$$

where $\hat{\mathbf{u}}_{c}^{0}=\left(\hat{\mathbf{u}}_{c, 1}^{0}, \hat{\mathbf{u}}_{c, 2}^{0}\right)$ is such that $\hat{u}_{c}(j)=K x(j)+$ $L \hat{y}_{s}^{0}(k)$ and $x(j+1)=(A+B K) x(j)+B L \hat{y}_{s}^{0}(k)$.

- Evaluate the costs $V_{t}\left(x(x+1), \tilde{\mathbf{u}}_{1}, \tilde{\mathbf{u}}_{2}\right)$ and $V_{t}(x(k+$ 1), $\left.\hat{\mathbf{u}}_{1}, \hat{\mathbf{u}}_{2}\right)$.

$$
\begin{aligned}
& \text { if }\left(x(k+1), \hat{y}_{s}^{0}(k)\right) \in \Omega_{t, K} \text { AND } \\
& V_{t}\left(x(k+1), \hat{\mathbf{u}}_{1}, \hat{\mathbf{u}}_{2}\right) \leq V_{t}\left(x(x+1), \tilde{\mathbf{u}}_{1}, \tilde{\mathbf{u}}_{2}\right) \text { then }
\end{aligned}
$$

Set

$$
\mathbf{v}(k+1)^{[0]}=\left(\hat{\mathbf{u}}_{1}(k+1), \hat{\mathbf{u}}_{2}(k+1), \hat{y}_{s}^{0}(k)\right)
$$

else

Set

$$
\mathbf{v}(k+1)^{[0]}=\left(\tilde{\mathbf{u}}_{1}(k+1), \tilde{\mathbf{u}}_{2}(k+1), \hat{y}_{s}^{0}(k)\right)
$$

\section{end if}

Remark 4. According to the algorithm, when the state of the system reaches the invariant set for tracking, that is $(x(k+$ 1), $\left.\hat{y}_{s}^{0}(k)\right) \in \Omega_{t, K}$, it is desiderable that the distributed MPC works better than the centralized terminal controller. If this is not possible, that is $V_{t}\left(x(k+1), \hat{\mathbf{u}}_{1}, \hat{\mathbf{u}}_{2}\right) \leq V_{t}(x(x+$ 1 ), $\tilde{\mathbf{u}}_{1}, \tilde{\mathbf{u}}_{2}$ ), hence the centralized terminal control law is chosen as warm start. With this choice convergence to the optimal centralized target and controllability of the solution are always ensured. This algorithm cannot be used in case of terminal equality constraint. In this case some controllability assumption would be necessary (Stewart et al. 2010).

Theorem 1. [Asymptotic stability] Consider $x(0) \in X_{N}$, where $X_{N}$ is the feasible set of problem (9). Then, for any $y_{t}$ the closed-loop system is asymptotic stable and converges to an equilibrium point $y_{s}$ such that

$$
y_{s}=\arg \min _{\hat{y}_{s} \in \lambda y_{s}} V_{O}\left(\hat{y}_{s}-y_{t}\right)
$$

Moreover, if $y_{t} \in \lambda y_{s}$, then $y_{s}=y_{t}$.

\subsection{Properties}

The proposed controller provides the following properties:

1 In what follows, the dependence of the variables from $x(k)$ will be omitted for the sake of clarity. 
- Enlargement of the domain of attraction. The domain of attraction of the MPC for regulation is the set of initial conditions that can be admissible steered to the optimal steady state $x_{s}$. For the proposed controller, the domain of attraction is the set of initial conditions that can be admissible steered to any steady state. Consequently, the domain of attraction of the proposed controller is (potentially) larger than the domain of the standard MPC.

- Changing operation points. Considering that the optimization problem is feasible for any $y_{t}$, then the proposed controller is able to track changing operation points maintaining the recursive feasibility and admissibility.

- Convergence to a centralized optimal equilibrium point. Since any agents solves an optimization problem with a centralized offset cost function and the warm start of any iteration is given by Algorithm 1, the proposed controller ensures convergence to the centralized optimal steady state, even in case of coupled constraints.

\section{EXAMPLE}

\subsection{Distributed model}

The presented controller has been tested in simulation on the system used for HD-MPC Benchmark, the 4 tanks system model.

The four tanks plant (Johansson 2000) is a multivariable laboratory plant of interconnected tanks with nonlinear dynamics and subject to state and input constraints. A scheme of this plant is presented in Figure 1(a). A real experimental plant developed at the University of Seville (Alvarado 2007) is presented in Figure 1(b). (a) Scheme of the 4 tank process.

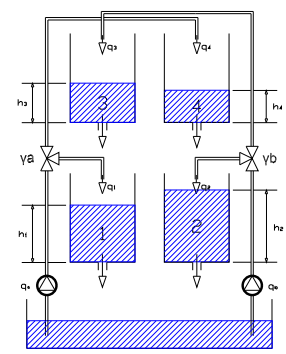

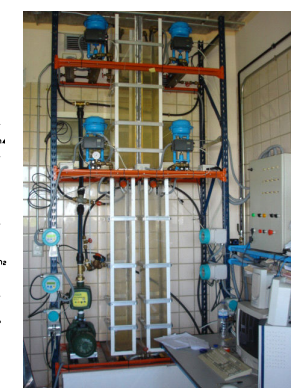

(b) The real plant.
Figure 1. The 4 tanks process.

A state space continuous time model of the quadruple tank process system is given in (Johansson 2000). Linearizing the model at an operating point given by $h_{i}^{0}$ and defining the deviation variables $x_{i}=h_{i}-h_{i}^{o}$ and $u_{j}=q_{j}-q_{j}^{o}$ where $j=a, b$ and $i=1, \cdots, 4$ we have that:

$$
\begin{aligned}
& \frac{d x}{d t}=\left[\begin{array}{cccc}
\frac{-1}{\tau_{1}} & 0 & \frac{A_{3}}{A_{1} \tau_{3}} & 0 \\
0 & \frac{-1}{\tau_{2}} & 0 & \frac{A_{4}}{A_{2} \tau_{4}} \\
0 & 0 & \frac{-1}{\tau_{3}} & 0 \\
0 & 0 & 0 & \frac{-1}{\tau_{4}}
\end{array}\right] x+\left[\begin{array}{cc}
\frac{\gamma_{a}}{A_{1}} & 0 \\
0 & \frac{\gamma_{b}}{A_{2}} \\
0 & \frac{\left(1-\gamma_{b}\right)}{A_{3}} \\
\frac{\left(1-\gamma_{a}\right)}{A_{4}} & 0
\end{array}\right] u \\
& y=\left[\begin{array}{llll}
1 & 0 & 0 & 0 \\
0 & 1 & 0 & 0
\end{array}\right] x
\end{aligned}
$$

where $\tau_{i}=\frac{A_{i}}{a_{i}} \sqrt{\frac{2 h_{i}^{0}}{g}} \geq 0, i=1, \cdots, 4$, are the time constants of each tank. This model has been discretized using the zeroorder hold method with a sampling time of 5 seconds.

The plant parameters, estimated on the real plant are shown in the following table:

\begin{tabular}{|l|c|l|l|}
\hline & Value & Unit & Description \\
\hline \hline$H_{1 \max }$ & 1.36 & $\mathrm{~m}$ & Maximum level of the tank 1 \\
$H_{2 \max }$ & 1.36 & $\mathrm{~m}$ & Maximum level of the tank 2 \\
$H_{3 \max }$ & 1.30 & $\mathrm{~m}$ & Maximum level of the tank 3 \\
$H_{4 \max }$ & 1.30 & $\mathrm{~m}$ & Maximum level of the tank 4 \\
$H_{\min }$ & 0.2 & $\mathrm{~m}$ & Minimum level in all cases \\
$Q_{a \max }$ & 3.6 & $\mathrm{~m}^{3} / h$ & Maximum flow of pump A \\
$Q_{b \max }$ & 4 & $\mathrm{~m}^{3} / h$ & Maximum flow of pump B \\
$Q_{\min }$ & 0 & $\mathrm{~m}^{3} / h$ & Minimal flow \\
$Q_{a}^{0}$ & 1.63 & $\mathrm{~m}^{3} / h$ & Equilibrium flow $\left(Q_{a}\right)$ \\
$Q_{b}^{0}$ & 2.0000 & $\mathrm{~m}^{3} / h$ & Equilibrium flow $\left(Q_{b}\right)$ \\
$a_{1}$ & $1.310 \mathrm{e}-4$ & $\mathrm{~m}^{2}$ & Discharge constant of tank 1 \\
$a_{2}$ & $1.507 \mathrm{e}-4$ & $\mathrm{~m}^{2}$ & Discharge constant of tank 2 \\
$a_{3}$ & $9.267 \mathrm{e}-5$ & $\mathrm{~m}^{2}$ & Discharge constant of tank 3 \\
$a_{4}$ & $8.816 \mathrm{e}-5$ & $\mathrm{~m}^{2}$ & Discharge constant of tank 4 \\
$A$ & 0.06 & $\mathrm{~m}^{2}$ & Cross-section of all tanks \\
$\gamma_{a}$ & 0.3 & & Parameter of the 3-ways valve \\
$\gamma_{b}$ & 0.4 & & Parameter of the 3-ways valve \\
$h_{1}^{0}$ & 0.6487 & $\mathrm{~m}$ & Equilibrium level of tank 1 \\
$h_{2}^{0}$ & 0.6639 & $\mathrm{~m}$ & Equilibrium level of tank 2 \\
$h_{3}^{0}$ & 0.6498 & $\mathrm{~m}$ & Equilibrium level of tank 3 \\
$h_{4}^{0}$ & 0.6592 & $\mathrm{~m}$ & Equilibrium level of tank 4 \\
\hline
\end{tabular}

The minimum level of the tanks has been taken greater than zero to prevent eddy effects in the discharge of the tank. One important property of this plant is that the dynamics present multivariable transmission zeros which can be located in the right hand side of the $s$ plane for some operating conditions. Hence, the values of $\gamma_{a}$ and $\gamma_{b}$ have been chosen in order to obtain a system with non-minimum phase multivariable zeros. In order to test the cooperative distributed MPC for tracking presented in the paper, the linear model has been partitioned in two subsystems in such a way that the two subsystems are interconnected through the inputs. The two subsystems model are the following:

$$
\begin{aligned}
& \frac{d x_{1}}{d t}=\left[\begin{array}{cc}
\frac{-1}{\tau_{1}} & \frac{A_{3}}{A_{1} \tau_{3}} \\
0 & \frac{-1}{\tau_{3}}
\end{array}\right] x_{1}+\left[\begin{array}{c}
\frac{\gamma_{a}}{A_{1}} \\
0
\end{array}\right] u_{1}+\left[\begin{array}{c}
0 \\
\frac{\left(1-\gamma_{b}\right)}{A_{3}}
\end{array}\right] u_{2} \\
& \frac{d x_{2}}{d t}=\left[\begin{array}{cc}
\frac{-1}{\tau_{2}} & \frac{A_{4}}{A_{2} \tau_{4}} \\
0 & \frac{-1}{\tau_{4}}
\end{array}\right] x_{2}+\left[\begin{array}{c}
0 \\
\frac{\left(1-\gamma_{a}\right)}{A_{4}}
\end{array}\right] u_{1}+\left[\begin{array}{c}
\frac{\gamma_{b}}{A_{2}} \\
0
\end{array}\right] u_{2} .
\end{aligned}
$$

where $x_{1}=\left(h_{1}, h_{3}\right), x_{2}=\left(h_{2}, h_{4}\right), u_{1}=q_{a}$ and $u_{2}=q_{b}$. The overall control objective is to control the level of tanks 1 and 2 while fulfilling the constraints on the levels and on the inputs.

\subsection{Simulations}

The controller has been tested in a simulation on the linearized system with four changes of reference. The starting points for agent 1 and 2 are $y_{1}=0.65$ and $y_{2}=0.65$ respectively. The references used are $y_{1, t}=(0.3,1.5,0.5,0.9)$ and $y_{2, t}=(0.3,1.5,0.75,0.75)$. The controllers' setups are: $\mathrm{N}=3$, $Q_{1,2}=I_{2}, R_{1,2}=0.01 I_{1}, w_{1,2}=0.5$. The gain $K$ is chosen 


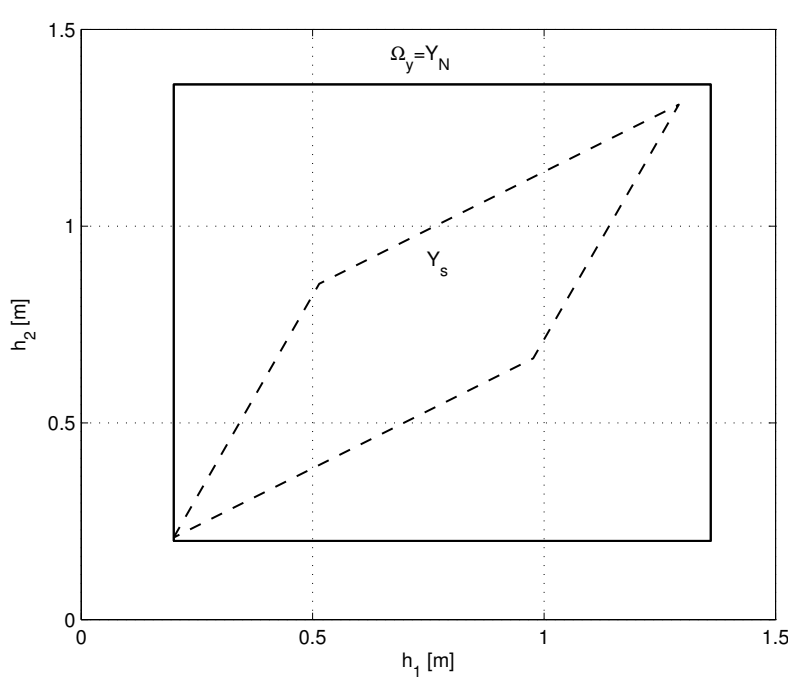

Figure 2. Steady output set, $y_{s}$, and projection of $\Omega_{t, K}$ onto $Y$, $\Omega_{t, Y}$.
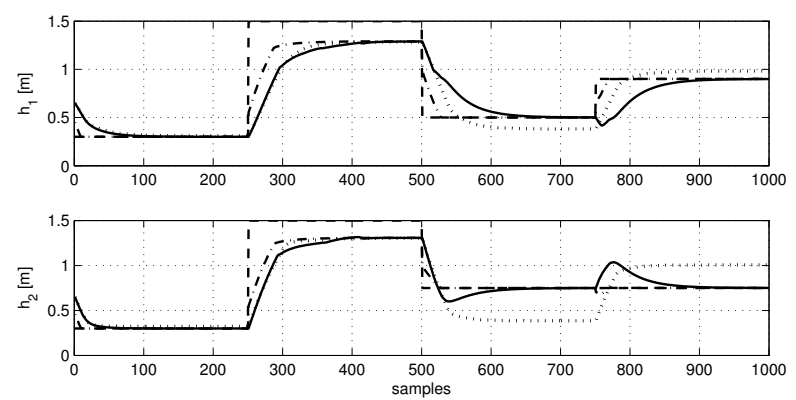

Figure 3. Time evolution of tanks 1 and 2 levels.

as the one of the LQR and the matrix $P$ is the solution of the Riccati equation.

The set of steady outputs of the system and the projection of the terminal constraints of the optimization problem $\Omega_{t, K}$, onto the admissible output space $Y$, are plotted in figure 2 . The time evolution of the levels $h_{1}$ and $h_{2}$ is plotted in Figure 3. The evolutions of the systems are plotted in solid lines, while the references and the artificial references are plotted respectively in dashed and dotted-dashed lines. See how the controller always steers the system to the given reference, and how the evolutions follow the artificial references while the real one are unfeasible. Notice, also, that in the second change of reference, when the target setpoint is unreachable (due to the constraints), the controller steers the system to the optimal steady state of the centralized problem.

The evolutions using the cooperative distributed MPC presented in (Rawlings and Mayne 2009, Chapter 6, pp. 456458 ), in case of a decentralized solution of the target problem, are plotted in dotted lines. See how this controller steers the system to a different steady state, given by the solution of a decentralized target problem.

\section{CONCLUSION}

In this paper a cooperative distributed linear model predictive control strategy has been proposed, applicable to any finite number of subsystems, for solving the problem of tracking nonzero setpoints. The proposed controller is able to steer the system to any admissible setpoint in an admissible way. Feasibility under any changing of the target steady state and convergence to the centralized optimal steady state are ensured. The proposed controller has been applied to a four tanks system.

\section{ACKNOWLEDGEMENTS}

This work has been funded by the European FP7 STREP project HD-MPC (FP7-ICT- 223854) and by MEC-Spain (contract DPI2008-05818) and by the U.S. NSF (\#CTS-0825306).

\section{REFERENCES}

Alvarado, I. (2007). Model Predictive Control for Tracking Constrained Linear Systems. Ph.D. thesis, Univ. de Sevilla.

Bemporad, A., Casavola, A., and Mosca, E. (1997). Nonlinear control of constrained linear systems via predictive reference management. IEEE Transactions on Automatic Control, 42, 340-349.

Camacho, E.F. and Bordons, C. (2004). Model Predictive Control. Springer-Verlag, 2nd edition.

Camponogara, E., Jia, D., Krogh, B.H., and Talukdar, S. (2002). Distributed Model Predictive Control. IEEE Control Systems Magazine, 22(1), 44-52.

Dunbar, W.B. (2007). Distributed Receding Horizon Control of Dynamically Coupled Nonlinear Systems. TAC, 52(7), 1249-1263.

Ferramosca, A., Limon, D., Alvarado, I., Alamo, T., and Camacho, E.F. (2009). MPC for tracking with optimal closed-loop performance. Automatica, 45, 1975-1978.

Gudi, R.D. and Rawlings, J.B. (2006). Identification for decentralized model predictive control. AIChE J, 52, 2198-2210.

Johansson, K.H. (2000). The quadruple-tank process. IEEE Trans. Cont. Sys. Techn., 8, 456-465.

Limon, D., Alvarado, I., Alamo, T., and Camacho, E. (2008). MPC for tracking of piece-wise constant references for constrained linear systems. Automatica, 44, 2382-2387.

Liu, J., Muñoz de la Peña, D., and Christofides, P.D. (2009). Distributed Model Predictive Control of Nonlinear Process Systems. AIChE Journal, 55(5), 1171-1184.

Liu, J., Muñoz de la Peña, D., Ohran, B.J., Christofides, P.D., and Davis, J.F. (2008). A two-tier Architecture for Networked Process Control. Chem. Eng. Sci., 63(22), 53945409.

Magni, L. and Scattolini, R. (2006). Stabilizing decentralized model predictive control for nonlinear systems. Automatica, 42(7), 1231-1236.

Mayne, D.Q., Rawlings, J.B., Rao, C.V., and Scokaert, P.O.M. (2000). Constrained model predictive control: Stability and optimality. Automatica, 36, 789-814.

Pannocchia, G. and Kerrigan, E. (2005). Offset-free reciding horizon control of constrained linear systems. AIChE Journal, 51, 3134-3146.

Raimondo, D.M., Magni, L., and Scattolini, R. (2007). Decentralized MPC of nonlinear systems: An input-to-state stability approach. Int. J. Robust Nonlinear Control, 17(17), 1651-1667.

Rao, C.V. and Rawlings, J.B. (1999). Steady states and constraints in model predictive control. AIChE Journal, 45, 1266-1278.

Rawlings, J.B. and Mayne, D.Q. (2009). Model Predictive Control: Theory and Design. Nob-Hill Publishing, 1st edition. 
Rossiter, J., Kouvaritakis, B., and Gossner, J. (1996). Guaranteeing feasibility in constrained stable generalized predictive control. IEEE Proc. Control theory Appl., 143, 463-469.

Sandell Jr., N.R., Varaiya, P., Athans, M., and Safonov, M. (1978). Survey of decentralized control methods for larger scale systems. IEEE Transactions on Automatic Control, 23(2), 108-128

Scokaert, P.O.M., Mayne, D.Q., and Rawlings., J.B. (1999) Suboptimal model predictive control (feasibility implies stability). IEEE Transactions on Automatic Control, 44(3), 648-654.

Stewart, B.T., Venkat, A.N., Rawlings, J.B., Wright, S.J., and Pannocchia, G. (2010). Cooperative distributed model predictive control. Systems \& Control Letters, 59, 460-469.

Venkat, A.N. (2006). Distributed Model Predictive Control: Theory and Applications. Ph.D. thesis, Univeristy of Wisconsin - Madison.

Venkat, A.N., Rawlings, J.B., and Wright, S.J. (2007). Distributed Model Predictive Control of Large-Scale Systems. Lecture Notes in Control and Information Sciences, 591605.

\section{Appendix A. TECHNICAL LEMMAS}

Lemma 5. [Recursive feasibility] Given a feasible initial solution $\left(\mathbf{u}_{1}^{[0]}(0), \mathbf{u}_{2}^{[0]}(0), \hat{y}_{s}^{[0]}(0)\right)$. Hence, $\forall p \geq 0$ and $k \geq 0$, the iterate $\left(\mathbf{u}_{1}^{[p]}(k), \mathbf{u}_{2}^{[p]}(k), \hat{y}_{s}^{[p]}(k)\right)$ is feasible.

Proof.

- Recursive feasibility of the iteration $p$.

Consider $k=0$ and $p=0$. Since $U$ and $\Omega_{t, K}$ are convex sets and $\left(\mathbf{u}_{1}^{0}\left(x, \mathbf{u}_{2}^{[0]}\right), \mathbf{u}_{2}^{[0]}, \hat{y}_{s, 1}^{[0]}\left(x, \mathbf{u}_{2}^{[0]}\right)\right)$ and $\left(\mathbf{u}_{1}^{[0]}, \mathbf{u}_{2}^{0}\left(x, \mathbf{u}_{1}^{[0]}\right), \hat{y}_{s, 2}^{[0]}\left(x, \mathbf{u}_{1}^{[0]}\right)\right)$ are feasible solutions, hence the convex combination of these solution is also feasible. Similarly, this is proved for any $p \geq 1$.

- Recursive feasibility of the time instant $k$.

Consider now the optimal solutions at time $k=0$, given by $\left(\mathbf{u}_{1}^{0}(0), \mathbf{u}_{2}^{0}(0), \hat{y}_{s}^{0}(0)\right)$, hence $\left(\mathbf{u}_{1}^{[0]}(1), \mathbf{u}_{2}^{[0]}(1), \hat{y}_{s}^{[0]}(1)\right)$ is a feasible solution for $x(1)$. If $\left(x(k+1), \hat{y}_{s}^{0}(k)\right) \in$ $\Omega_{t, K}$ and $V_{t}\left(x(k+1), \hat{\mathbf{u}}_{1}, \hat{\mathbf{u}}_{2}\right) \leq V_{t}\left(x(x+1), \tilde{\mathbf{u}}_{1}, \tilde{\mathbf{u}}_{2}\right)$, the centralized terminal control law provides a feasible solution. Otherwise, the first $N-1$ terms of $\tilde{\mathbf{u}}_{1}$ and $\tilde{\mathbf{u}}_{2}$ are feasible and the last one, which is the centralized terminal control law is feasible as well. Similarly, this is proved for any $k>0$.

Lemma 6. [Convergence of the algorithm] For any $k \geq$ 0 and $p \geq 0, V_{t}\left(x(k), \mathbf{u}_{1}^{[p+1]}(k), \mathbf{u}_{2}^{[p+1]}(k), \hat{y}_{s}^{[p+1]}(k)\right) \leq$ $V_{t}\left(x(k), \mathbf{u}_{1}^{[p]}(k), \mathbf{u}_{2}^{[p]}(k), \hat{y}_{s}^{[p]}(k)\right)$

Proof. Removing the time dependence for the sake of simplicity, we have that:

$$
\begin{aligned}
V_{t}\left(x, \mathbf{u}_{1}^{[p+1]}, \mathbf{u}_{2}^{[p+1]}, \hat{y}_{s}^{[p+1]}\right) \leq & w_{1} V_{t}\left(x, \mathbf{u}_{1}^{0}\left(x, \mathbf{u}_{2}^{[p]}\right), \mathbf{u}_{2}^{[p]}, \hat{y}_{s, 1}^{[p]}\left(x, \mathbf{u}_{2}^{[p]}\right)\right) \\
& +w_{2} V_{t}\left(\mathbf{u}_{1}^{[p]}, \mathbf{u}_{2}^{0}\left(x, \mathbf{u}_{1}^{[p]}\right), \hat{y}_{s, 2}^{[p]}\left(x, \mathbf{u}_{1}^{[p]}\right)\right) \\
\leq & w_{1} V_{t}\left(x, \mathbf{u}_{1}^{[p]}, \mathbf{u}_{2}^{[p]}, \hat{y}_{s}^{[p]}\right) \\
& +w_{2} V_{t}\left(x, \mathbf{u}_{1}^{[p]}, \mathbf{u}_{2}^{[p]}, \hat{y}_{s}^{[p]}\right) \\
= & V_{t}\left(x, \mathbf{u}_{1}^{[p]}, \mathbf{u}_{2}^{[p]}, \hat{y}_{s}^{[p]}\right)
\end{aligned}
$$

The first inequality comes from convexity of the solution, and the second one from feasibility and optimality of the solution.
Lemma 7. [Local bounding] Let $k$ be an instant such that $\left(x(k), \hat{y}_{s}^{[0]}(k)\right) \in \Omega_{t, K}$. Then $V_{t}\left(x(k), \mathbf{u}_{1}^{[p]}(k), \mathbf{u}_{2}^{[p]}(k), \hat{y}_{s}^{[p]}(k)\right)$ $\leq\left\|x(k)-\hat{x}_{s}^{[0]}(k)\right\|_{P}^{2}+V_{O}\left(\hat{y}_{s}^{[0]}(k)-y_{t}\right)$

Proof. If $\left(x(k), \hat{y}_{s}^{[0]}(k)\right) \in \Omega_{t, K}$, hence the initialization of the algorithm ensures that $V_{t}\left(x(k), \mathbf{u}_{1}^{[0]}(k), \mathbf{u}_{2}^{[0]}(k), \hat{y}_{s}^{[0]}(k)\right) \leq$ $\left\|x(k)-\hat{x}_{s}^{[0]}(k)\right\|_{P}^{2}+V_{O}\left(\hat{y}_{s}^{[0]}(k)-y_{t}\right)$. This fact and lemma 6 prove the lemma.

Lemma 8. Let $y_{\infty}$ and a time instant $k$ such that, $x_{\infty}=M_{x} y_{\infty}$, $u^{+}(j ; k)=M_{u} y_{\infty}$ and $\hat{y}_{s}(k)=y_{\infty}$. Then, $V_{t}^{0}\left(x_{\infty}\right)=$ $V_{O}\left(y_{\infty}-y_{t}\right)$

Proof. It is clear that $y_{\infty}$ is a fixed point for the closed-loop system. At time $\mathrm{k}: x(k)=x_{\infty}, u(k)=u_{\infty}, \hat{y}_{s}(k)=y_{\infty}$. This implies that $x(k+1)=x_{\infty}$ and $\hat{y}_{s}^{[0]}(k+1)=y_{\infty}$, and hence $\left(x(k+1), \hat{y}_{s}^{[0]}(k+1)\right) \in \Omega_{t, K}$. Hence

$$
\begin{aligned}
V_{t}^{0}(x(k+1)) & \leq V_{t}\left(x(k+1), \mathbf{u}_{1}^{[0]}(k+1), \mathbf{u}_{2}^{[0]}(k+1), \hat{y}_{s}^{[0]}(k+1)\right) \\
& \leq\left\|x(k+1)-M_{x} y_{s}^{[0]}(k+1)\right\|_{P}^{2}+V_{O}\left(\hat{y}_{s}^{[0]}-y_{t}\right)
\end{aligned}
$$

Since $x(k+1)=x_{\infty}=M_{x} y_{\infty}=M_{x} y_{s}^{[0]}(k+1)$, hence $V_{t}^{0}\left(x_{\infty}\right) \leq V_{0}\left(y_{\infty}-y_{t}\right)$.

Let $V_{N}^{0}\left(x_{\infty}\right)$ be the optimal centralized solution taking $\hat{y}_{s}=$ $y_{\infty}$. Then, $V_{N}^{0}\left(x_{\infty}\right)=V_{O}\left(y_{\infty}-y_{t}\right)$. Hence, since $y_{\infty}$ is a fixed point, $\hat{y}_{s}^{0}(k+1)=y_{\infty}$. Therefore, $\left(\mathbf{u}_{1}^{0}(k+1), \mathbf{u}_{2}^{0}(k+1)\right)$ is a suboptimal solution of the centralized problem, and hence $V_{t}^{0}(x(k+1)) \geq V_{N}^{0}(x(k+1))=V_{N}\left(x_{\infty}\right)=V_{O}\left(y_{\infty}-y_{t}\right)$ which proves the lemma.

\section{Appendix B. PROOF OF THEOREM 1}

Proof. Given the initial solution in $x(0), \mathbf{u}_{1}^{[0]}(0)$ and $\mathbf{u}_{2}^{[0]}(0)$, lemma 5 ensures that $\mathbf{u}_{1}^{0}(k)$ and $\mathbf{u}_{2}^{0}(k)$ are admissible and moreover $x(k) \in X$ for any $k$.

From lemma 6:

$$
\begin{aligned}
\Delta V_{t}= & V_{t}^{0}(x(k+1))-V_{t}^{0}(x(k)) \\
\leq & V_{t}\left(x(k+1), \mathbf{u}_{1}^{[0]}(k+1), \mathbf{u}_{2}^{[0]}(k+1), \hat{y}_{s}^{[0]}(k+1)\right)-V_{t}^{0}(x(k)) \\
\leq & V_{t}\left(x(k+1), \tilde{\mathbf{u}}_{1}(k+1), \tilde{\mathbf{u}}_{2}(k+1), \tilde{y}_{s}(k+1)\right)-V_{t}^{0}(x(k)) \\
\leq & -\left\|x(k)-\hat{x}_{s}^{0}(k)\right\|_{Q}^{2}-\left\|u_{1}(k)-\hat{u}_{s, 1}^{0}(k)\right\|_{R_{1}}^{2} \\
& -\left\|u_{2}(k)-\hat{u}_{s, 2}^{0}(k)\right\|_{R_{2}}^{2}
\end{aligned}
$$

Given that the cost function is a positive definite function:

$\lim _{k \rightarrow \infty}\left|x(k)-x_{\infty}\right|=0, \lim _{k \rightarrow \infty}\left|u(k)-u_{\infty}\right|=0, \lim _{k \rightarrow \infty}\left|\hat{y}_{s}(k)-y_{\infty}\right|=0$ and $x_{\infty}=M_{x} y_{\infty}$ and $u_{\infty}=M_{u} y_{\infty}$. By continuity, we can state that the system converges to a fixed point.

Hence, using lemma $8, V_{t}^{0}\left(x_{\infty}\right)=V_{O}\left(y_{\infty}-y_{t}\right)$. Using same arguments as in (Ferramosca et al. 2009, Theorem 1), we can state that $y_{\infty}$ is the minimizer of $V_{O}\left(\hat{y}_{s}-y_{t}\right)$ and then $M_{x} y_{\infty} \in$ $X$ and $M_{u} y_{\infty} \in U$. Moreover, if $y_{t}$ is such that $M_{x} y_{t} \in X$ and $M_{u} y_{t} \in U$, then $y_{\infty}=y_{t}$. 